\title{
Time-Dependent Surface Impedance From Sferics
}

\author{
Yvette O'Keefe, Student Member, IEEE, David V. Thiel, Senior Member, IEEE, and Steven G. O'Keefe, Member, IEEE
}

\begin{abstract}
Attempts to use discrete sferics for low-frequency impedance mapping have relied on Fourier analysis to derive time-independent frequency profiles of the earth. A one-dimensional transmission line model of the earth above a perfectly conducting layer is used to demonstrate that the impedance is time dependent. Assuming a sferic-type transient for the applied magnetic field, the electric field is changed significantly corresponding to the time of flight of the radiation in the lossy earth. In an attempt to maintain this timing information and its link with the frequency components, the wavelet transform was implemented to analyze the data. The associated surface impedance may be approximated as the input impedance of the earth during excitation but reverts to the impedance of free space once the source field returns to zero.
\end{abstract}

Index Terms-Sferics, surface impedance, transmission line model, wavelets.

\section{INTRODUCTION}

A TMOSPHERICS (sferics) are the most dominant natural source electromagnetic (EM) waves in the extremely low frequency (ELF) very low frequency (VLF) range propagating in the earth ionosphere waveguide. The conductivity and permittivity of the earth and their distribution at the surface or any existing subsurface layers influence the relationship between these propagating fields [1], [2]. It is this influence that provides a mechanism for passive geophysical investigation at a measurement site. This EM geophysical survey technique is referred to as the surface impedance method, and it encompasses magnetotelluric (MT), audio-magnetotelluric (AMT), and lightning-based techniques as well as artificial source methods such as controlled source audio-magnetotelluric (CSAMT) and VLF [3]. The technique of interest is the lightning-based method referred to as VLF-MT [4].

Cloud-to-ground lightning sferics are regarded as noise in the other techniques and are filtered out. VLF-MT takes advantage of the broadband frequency spectra present in these transient events. The desired signals originating in the far field present as propagating transverse magnetic (TM) plane waves [5]. Garner and Thiel have shown that these signals provide interpretable surface impedance data [6]. Hill and Thiel extended this work replacing the Fourier analysis with the wavelet transform, thus removing the necessity of collecting large amounts of data and the required statistical manipulation to obtain greater accuracy [7]. The wavelet analysis, however, provides time domain impedance information, a view now explored in this letter.

Manuscript received November 7, 2004; revised December 9, 2004.

The authors are with the Centre for Wireless Monitoring and Applications, Griffith University, Nathan, Queensland, Australia (e-mail: y.okeefe@ griffith.edu.au; d.thiel@griffith.edu.au; s.okeefe@griffith.edu.au).

Digital Object Identifier 10.1109/LGRS.2004.843206

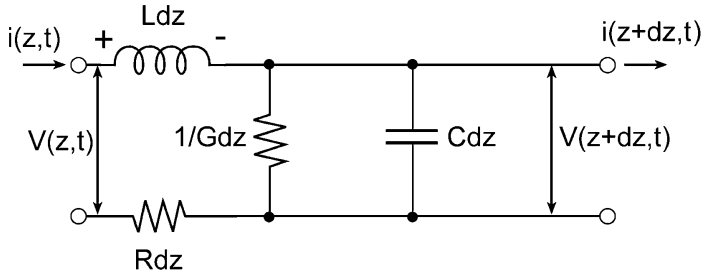

Fig. 1. Single transmission line cell used to model propagation in the earth and atmosphere.

\section{TRANSMISSION LINE MODEL}

The propagation of a normally incident plane wave in a lossy medium is analogous to propagation in a transmission line [8], [9]. Hence, the characteristic impedance $Z_{0}$ of the medium or line can be determined using either the electrical properties of the earth or the electrical components of the line, as in (1). $R, L$, $G$, and $C$ are the resistance, inductance, conductance, and capacitance per unit length of the transmission line, respectively. $\gamma_{0}, \sigma_{0}, \varepsilon_{0}$ are the characteristic propagation constant, conductivity, and permittivity

$$
Z_{0}=\sqrt{\frac{R+j \omega L}{G+j \omega C}}=\frac{\gamma_{o}}{\left(\sigma_{0}+j \varepsilon_{0} \omega\right)} .
$$

The RLC circuit for a single cell in a transmission line model (TLM) is shown in Fig. 1, where $d z$ represents the length of the cell and the distance in the propagating medium. A lumped RLC network used in [10] was selected to model two homogeneous half spaces representing an air-earth interface. This network is representative of a one-dimensional TLM where $V(z, t)$ and $I(z, t)$ are the voltage and current. The air-medium interface is located at $z=0$. The Fourier transform of the voltage $V(\omega)$ and current $I(\omega)$ at the interface are equivalent to the transformed electric $\left(E_{x}\right)$ and magnetic $\left(H_{y}\right)$ fields, respectively. These fields are used to calculate the surface impedance $Z_{s}(\omega)$ or input impedance and can be determined by

$$
Z_{s}(\omega)=\frac{E_{x}(\omega)}{H_{y}(\omega)}=\frac{V(\omega)}{I(\omega)}=Z_{0} \frac{Z_{0} \tanh \left(\gamma_{1} l_{1}\right)+Z_{L}}{Z_{L} \tanh \left(\gamma_{1} l_{1}\right)+Z_{0}} .
$$

$E_{x}$ is the horizontal electric field component of the radio wave measured in the plane of incidence, and $H_{y}$ is the horizontal magnetic field measured perpendicular to the plane of incidence. The termination impedance at the end of the line is $Z_{L}$, and $l_{1}$ and $\gamma_{1}$ are the length and propagation constant of the line representing layer one, with $\gamma_{1}$ reducing to the following when $R=0$ :

$$
\gamma_{1}=\sqrt{(R+j \omega L)(G+j \omega C)}= \pm \sqrt{j \omega \mu(\sigma+j \omega \varepsilon)} .
$$


TABLE I

ELECTRICAL PROPERTIES USED IN THE MODEL

\begin{tabular}{l|l|l|l}
\hline & $\begin{array}{l}L=\mu \\
(\mu \mathrm{H} / \mathrm{m})\end{array}$ & $\begin{array}{l}G=\sigma \\
(\mathrm{S} / \mathrm{m})\end{array}$ & $\begin{array}{l}C=\varepsilon_{o} \varepsilon_{r} \\
(\mathrm{pF} / \mathrm{m})\end{array}$ \\
\hline Air & 1.2566 & 0 & 8.85 \\
\hline Earth & 1.2566 & $1 \mathrm{e}-5$ & 88.5 \\
\hline
\end{tabular}

As shown in [10], the following substitutions may be made, $L \equiv \mu, G \equiv \sigma, C \equiv \varepsilon$, where $\mu, \sigma, \varepsilon$ are the permeability, conductivity, and permittivity of the media respectively.

The TLM circuit was modeled with Micro-Cap 8 electrical circuit simulation software (Spectrum Software, Sunnyvale, CA), which avoids the irregular time stepping problems that other PSPICE-based simulators suffer when exposed to short transient events. The software also provides a mechanism to introduce an arbitrary waveform, being any number of prerecorded magnetic $\mathrm{H}$ field vectors as the signal source.

To simplify the circuit and limit the number of components required, a shunt resistance equivalent to the intrinsic impedance of air $\left(\eta_{0}=120 \pi \Omega\right)$ represents the circuit model for the air half space. Each cell in the model is equivalent to an RLC cell and represents a particular propagation distance in the medium dependent on the value of $d z$. The model used five and ten cells with $d z$ equivalent to 200 or $100 \mathrm{~m}$, respectively. This is equivalent to a propagation distance through an homogenous layer of depth $1000 \mathrm{~m}$. Termination at the end of the line is completed with a short circuit (equivalent to a perfectly conducting basement), an open circuit (a perfectly resistive basement), or a matched impedance (an infinitely deep material).

Two physical models were investigated: the first examined propagation in air alone to verify the method, and the second represented propagation toward an air-earth interface. In order to include the low propagation velocities at low frequencies, the TLM representing the air model was a 20-cell network representing a depth of $2000 \mathrm{~m}$. This is twice the distance used in the air-earth interface model. The electrical properties used in both simulations are given in Table I. As $G$ is zero in air, each cell consists only of an inductor and capacitor.

The surface impedance of both models was calculated using two different methods: a standard Fourier transform from the time to the frequency domain of the voltage and current, and a wavelet transformation, which provides both frequency and time information.

\section{A. Wavelet Transformation}

Using a discrete dataset for both current and voltage, a discretized version of the continuous wavelet transform $\hat{p}(\alpha, \beta)$ defined by the following was used:

$$
\hat{p}(\alpha, \beta)=\frac{1}{\sqrt{\alpha}} \int_{-\infty}^{\infty} p(t) w\left(\frac{t-\beta}{\alpha}\right) d t
$$

where $p(t)$ represents both $V(0, t)$ and $I(0, t)$. The scaling function $w_{\alpha, \beta}(t)$, with the scaling factor $\alpha$ and translation $\beta$ is given in

$$
w_{\alpha, \beta}(t)=\frac{1}{\sqrt{\alpha}} w\left(\frac{t-\beta}{\alpha}\right) .
$$
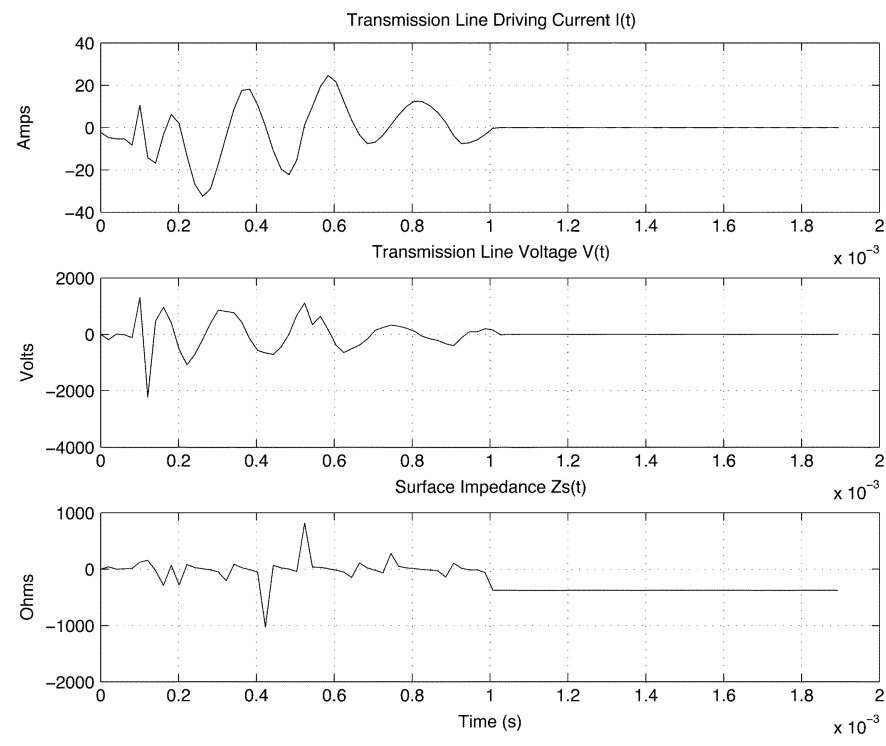

Fig. 2. Transmission line model with driving current $I(t)$, voltage $V(t)$, and surface impedance $Z s(t)$ measured across a 1000 -m air-earth model transmission line terminated with a short circuit.

The basis set is developed from the scaling function where each dilation is controlled by $\alpha$, which is indirectly proportional to $\omega_{0}$, the dilation's centre angular frequency. The power of the mother wavelet is maintained by $1 / \sqrt{ } \alpha$, in each dilation. The shift in the time domain results from each translation.

Computation time was minimized by performing the transform using convolution theory and the fast Fourier transform given in

$$
\hat{p}(\alpha, \beta)=\frac{1}{\sqrt{2 \pi \alpha}} \int_{-\infty}^{\infty} W(\alpha, \omega) P(\omega) e^{j \beta \omega} d \omega .
$$

$W(\alpha, \omega)$ and $P(\omega)$ are the Fourier transform of the scaling function $w_{\alpha, \beta}(t)$ and the raw data $p(t)$, respectively.

The surface impedance is calculated by substituting the wavelet coefficients of both the voltage and current into

$$
Z_{s}\left(\omega_{0}, t\right)=\frac{\hat{v}(\alpha, \beta)}{\hat{i}(\alpha, \beta)}
$$

where $\hat{v}(\alpha, \beta)$ and $\hat{i}(\alpha, \beta)$ are the coefficients of the signals as a function of dilation $\alpha$ and translation $\beta$.

The surface impedance $Z_{s}$ is a function of frequency $\omega_{\mathrm{o}}$ and time $t$, where $\omega_{0}$ represents the center angular frequency of each dilation. A detailed proof of the above procedure and a detailed discussion regarding the benefits of the wavelet transform (WT) for MT processing as opposed to the windowed Fourier transform may be found in [11].

\section{RESULTS}

A previously recorded far-field measurement of an $\mathrm{H}$ field cloud-to-ground lightning strike was used to provide the current for the model (see Fig. 2). As most of the power in the sferic had dissipated after $1 \mathrm{~ms}$, the recording was windowed for this period. The recorded signal from the simulation, however, was allowed to continue for a further $0.8 \mathrm{~ms}$, thus providing a mechanism to monitor any reflections present. 


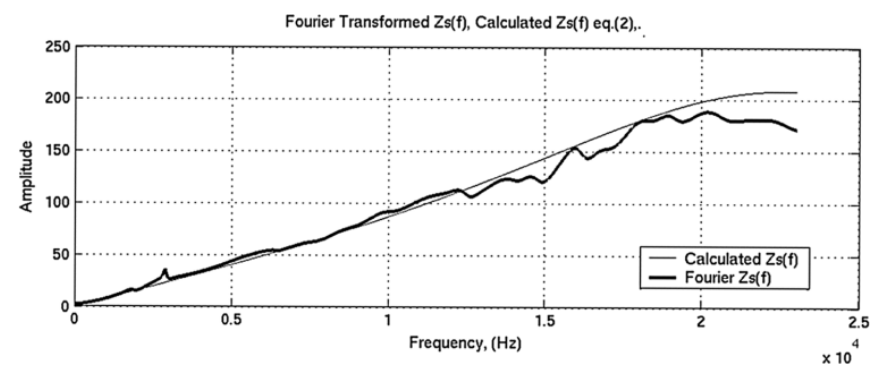

(a)

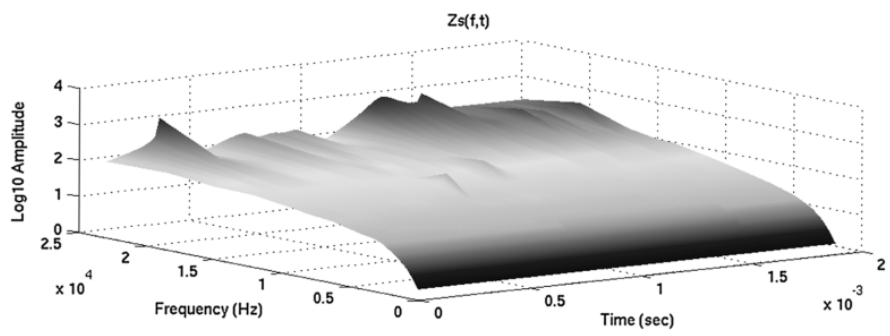

(b)

Fig. 3. (a) Measured and calculated Fourier surface impedance $Z s(f)$. (b) The surface impedance $Z s(f, \mathrm{t})$ in the wavelet domain measured across a $1000-\mathrm{m}$ air-earth model with a perfectly conducting basement.

\section{A. Air Model}

When a matched impedance was used to terminate the air transmission line, the measured surface impedance was found to be equal to the intrinsic impedance of air $\eta_{0}$. This value was constant for all frequencies and times. Due to the lossless nature of the structure, the voltage and current waveforms are identical in shape. As expected, no reflections occur at either the interface or the termination; hence, the model represents an infinite line with the intrinsic impedance of air.

The termination was changed first to an open circuit and then to a short circuit. The impedance mismatch causes reflected signals to propagate back to the source. The sum of incident and reflected signals changes with time as the amplitude of the source signal changes with time. The surface impedance at this point is, therefore, time dependent. If signals reflect from the source, then further impedance changes occur at later times.

The surface impedance was obtained from the model using both a Fourier and wavelet transform. The Fourier transform gives the impedance as a function of frequency. The wavelet transform gives impedance versus frequency and time and requires a three-dimensional plot. Normally the surface impedance described by (2) is expected, but as a sferic signal is used for the source, the surface impedance was seen to deviate from this function during the period of the sferic. This is, of course, only observable on the wavelet transform. The deviation in impedance is most noticeable at higher frequencies (above $15 \mathrm{kHz}$ ), as the model depth is approaching a quarter wavelength at these frequencies. After the sferic driving signal finishes, the high-frequency impedance value assumes the value of the source impedance, in this case $\eta_{0}$. This is because the signals are now only propagating out of the model. At low frequencies, the surface impedance approaches the impedance of the termination.

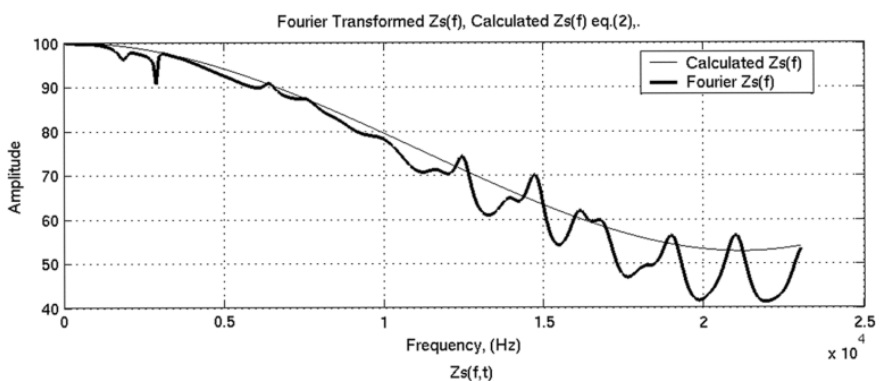

(a)



(b)

Fig. 4. (a) Measured and calculated Fourier surface impedance $Z s(f)$. (b) The surface impedance $Z s(f, t)$ in the wavelet domain measured across a 1000-m air-earth model with an insulating basement (open circuit termination).

\section{B. Air-Earth Model: Perfectly Conductive Basement}

The air-earth model (earth conductivity $\sigma=10 \mu \mathrm{S} / \mathrm{m}$, permittivity $\varepsilon_{r}=10$ ) was terminated with a short-circuit termination. The source time-domain signals (voltage $V(t)$, current $I(t)$, and impedance $Z(t))$ are shown in Fig. 2 .

It can clearly be seen that $Z(t)$ returns to $\eta_{o}$ once the driving signal has terminated. As the propagation direction is away from the earth's surface, the impedance is negative. In Fig. 2, note that the reflected signals have changed the shape of $V(t)$ when compared to driving current $I(t)$.

The surface impedance calculated using (2) is compared with the Fourier transform surface impedance $Z_{s}(f)$ and is illustrated in Fig. 3(a). Fig. 3(b) shows the three-dimensional wavelet transform surface impedance $\mathrm{Z}(f, t)$. Both methods show that the surface impedance trends along the calculated impedance from (2). However, it can be observed that at higher frequencies, the Fourier and wavelet impedances deviate and tend to oscillate. These deviations are produced by changes in the amplitude and phase of individual frequencies of the driving current. When the forward and reflected signals interact, a different value is obtained because the reflected signal has been delayed by the propagation time. These variations in the voltage and current measured at the surface result in a change in the impedance. Reflected signals will also be rereflected from the air-earth interface and will contribute again as they propagate to the termination and back again. These impedance deviations are larger for signal wavelengths that are shorter than the model depth.

The wavelet transform gives further information in that the impedance may be observed during and after the driving sferic signal has been injected. Again, the impedance variations due to the structure are most noticeable in the high frequencies. While the sferic signal is present, the surface impedance is directly related to the incident and reflected signals. After the sferic has 
finished, the impedance is related to reflected signals only. The strongest of these is the signal propagating out of the earth. The impedance is, therefore, mainly representative of the air over the earth. This is evident in Fig. 3(b), as a high-impedance plateau at high frequencies after $t=1 \mathrm{~ms}$.

\section{Air-Earth Model: Perfectly Insulating Basement}

The results of the air-earth model with a perfectly insulating basement (open circuit termination) are shown in Fig. 4. In Fig. 4(a), the calculated and modeled Fourier surface impedances $Z_{s}(f)$ are compared. In Fig. 4(b), the wavelet surface impedance $Z(f, t)$ is shown as a surface plot. Both methods again show reasonable correlation with the calculated values except in the higher frequency range. Between $10 \mathrm{k}$ and $23 \mathrm{kHz}$, the Fourier impedance shows a cyclic variance in the frequency domain from that calculated. This effect is again caused by variations in the sferics spectral amplitudes with time. This causes the surface impedance to vary with time due to the delayed reflections summating with the sferic signal. There is also a contribution from reflections that have been rereflected from the source. The wavelet transform result shows more clearly the impedance variation with time of the sferic and represents the impedance looking into the earth. When the sferic has finished, the impedance value represents the impedance looking out of the earth, i.e., $\eta_{0}$. This is again evident as a plateau in the surface plot in Fig. 4(b) for high frequencies after $1 \mathrm{~ms}$.

\section{CONCLUSION}

It has been demonstrated that a measure of the wavelet-based surface impedance of the earth using a time-varying sferic signal may lead to the collection of more informative data. The Fourier method yields the surface impedance versus frequency. This impedance, however, has been shown to be time dependent, and so the Fourier technique is inaccurate at higher frequencies. If the time-dependent impedance is considered, additional information about the earth's conductivity profile can be obtained. This is due to the spectral power variations in the sferic and the delay time and reflection coefficients for signals arriving back at the surface. A windowed Fourier transform method could be employed, but these methods typically result in poor resolution [12]. If the wavelet transform method is employed, further information regarding the impedance mainly due to the sferic traveling into the earth or reflected signals traveling toward the surface of the earth may be obtained. This work suggests that inversion techniques should be able to use the surface impedance as a function of time and frequency to more uniquely identify subsurface structures.

Further investigations are planned with two- and three-dimensional earth models. Effective inversion techniques for the sferic data are also under consideration.

\section{REFERENCES}

[1] K. Vozoff, "The magnetotelluric method," in Electromagnetic Methods in Applied Geophysics, M. N. Nabighian, Ed. Tulsa, OK: Soc. Explor. Geophys., 1991, vol. 2B, pp. 641-711.

[2] J. R. Wait, "Influence of finite ground conductivity on the fields of a vertical travelling wave of current," IEEE Trans. Electromagn. Compat., vol. 41, no. 1, p. 78, Jan. 1999.

[3] IEEE, "IEEE guide for measurements of electromagnetic properties of earth media," IEEE, Piscataway, NJ, IEEE STD 356, 2001.

[4] S. J. Garner, "Broadband surface impedance measurements using VLF spherics and artificial HF," Ph.D. thesis, School of Microelectron. Eng., Griffith Univ., Brisbane, Australia, 1998.

[5] J. R. Wait, Geo-Electromagnetism. Sydney, Australia: Academic, 1982, p. 184.

[6] S. J. Garner and D. V. Thiel, "Broadband (ULF-VLF) surface impedance measurements using MIMDAS," Explor. Geophys., vol. 31, pp. 173-178, 2000.

[7] Y. Hill, D. V. Thiel, and S. G. O'Keefe, "Wavelets-A new approach to surface impedance measurement using radiation from lightning," in Proc. Bowen Basin Symp. 2000, 2000, pp. 249-252.

[8] D. K. Cheng, Field and Wave Electromagnetics. Reading, MA: Addison-Wesley, 1989, pp. 440-441.

[9] A. H. Zemanian, "Operator-valued transmission lines as models of a horizontally layered earth under transient two-dimensional electromagnetic excitation," IEEE Trans. Antennas Propag., vol. AP-33, no. 3, pp. 345-350, Mar. 1985.

[10] T. P. Weldon, Y. A. Gryazin, and M. V. Kilbanov, "Comparison of 2D and 1D approaches to forward problem in mine detection," Proc. SPIE, vol. 4038, pp. 1140-1148, 2000.

[11] Y. Zhang, D. Goldak, and K. Paulson, "Detection and processing of lightning-sourced magnetotelluric transients with the wavelet transform," IEICE Trans. Fund., vol. E80-A, no. 5, pp. 849-558, 1997.

[12] J. S. Walker, A Primer on Wavelets and Their Scientific Applications. Boca Raton, FL: CRC, 1999. 